\title{
Response time is more important than walking speed for the ability of older adults to avoid a fall after a trip
}

\author{
A.J. van den Bogert*, M.J. Pavol, M.D. Grabiner \\ Department of Biomedical Engineering, ND-2, The Cleveland Clinic Foundation, 9500 Euclid Avenue, Cleveland, OH 44195, USA \\ Accepted 19 September 2001
}

\begin{abstract}
We previously reported that the probability of an older adult recovering from a forward trip and using a "lowering" strategy increases with decreased walking velocity and faster response time. To determine the within-subject interaction of these variables we asked three questions: (1) Is the body orientation at the time that the recovery foot is lowered to the ground ("tilt angle") critical for successful recovery? (2) Can a simple inverted pendulum model, using subject-specific walking velocity and response time as input variables, predict this body orientation, and thus success of recovery? (3) Is slower walking velocity or faster response time more effective in preventing a fall after a trip? Tilt angle was a perfect predictor of a successful recovery step, indicating that the recovery foot placement must occur before the tilt angle exceeds a critical value of between $23^{\circ}$ and $26^{\circ}$ from vertical. The inverted pendulum model predicted the tilt angle from walking velocity and response time with an error of $0.4 \pm 2.2^{\circ}$ and a correlation coefficient of 0.93 . The model predicted that faster response time was more important than slower walking velocity for successful recovery. In a typical individual who is at risk for falling, we predicted that a reduction of response time to a normal value allows a $77 \%$ increase in safe walking velocity. The mathematical model produced patient-specific recommendations for fall prevention, and suggested the importance of directing therapeutic interventions toward improving the response time of older adults. (C) 2002 Elsevier Science Ltd. All rights reserved.
\end{abstract}

Keywords: Falling; Prevention; Simulation; Aging; Locomotion

\section{Introduction}

Falls and fall-related injuries are among the "most serious and common medical problems experienced by the elderly" (Hayes et al., 1996). In the United States there are approximately 300,000 hip fractures per year in older adults, of which $90 \%$ result from falls. Thirtythree percent of women and $17 \%$ of men will fracture a hip if they live to 90 years of age. In older adults who have experienced a hip fracture the mortality rate is up to $20 \%$ higher than in older adults of similar age, sex, and race (Hayes et al., 1996). Tripping is a major contributor to falls and fall-related injuries, particularly hip fractures, in older adults. For example, of 125 hip fractures for which a detailed fall history was available,

\footnotetext{
*Corresponding author. Tel.: +1-216-444-5566; fax: + 1-216-4449198.

E-mail address: bogert@bme.ri.ccf.org (A.J. van den Bogert).
}

$89 \%$ occurred as a result of a fall and $38 \%$ occurred as a result of a trip (Cumming and Klineberg, 1994). In another study, of 123 hip fractures for which a detailed fall classification was acquired, $95 \%$ were the result of a fall and $12 \%$ were the result of a trip (Nyberg et al., 1996).

Walking velocity is one of the many variables that have been associated with falls by older adults. Older adults, particularly frail older adults who walk slowly have a significantly higher risk of falling (Bath and Morgan, 1999) and higher risk for hip fracture (Dargent-Molina et al., 1996). However, it is not clear whether slow walking velocity contributed to injury risk, or was a protective adaptation to compensate for other risk factors. Walking velocity also influences the biomechanics of fall descent and, therefore, may indirectly influence the fracture site. For example, Smeesters et al. (2001) reported that slow walking velocity increases the probability of an impact on or near the greater trochanter after a trip. 
We developed an experimental protocol to induce unexpected trips in older adults during locomotion. Using this protocol, we have characterized the mechanisms underlying the diminished capacity to execute appropriate stepping responses (Pavol et al., 1999a, b, 2001). One particular category of older adults, defined by their recovery strategy (lowering) and fall type (during step) was discriminated by a significantly faster walking velocity at the instant of the trip and by a significantly slower response time in response to the trip. These results suggest that the likelihood of recovery following a trip may be increased if walking velocity is reduced and/or response time is decreased. From the standpoint of therapeutic intervention, it is easier to influence walking velocity than response time. Nevertheless, the extent to which each of these variables individually contribute to the outcome is not known.

Walking velocity contributes to the speed of forward rotation of the body after the trip, while response time determines the duration of this rotation before recovery is initiated. Both variables thus contribute to the body orientation at the time that the tripped foot contacts the ground. It is possible that a critical body orientation exists beyond which it is too late to initiate recovery. If this is the mechanism by which walking velocity and response time affect success of recovery, it should be possible to determine theoretically the relative influences of each on the risk of falling after a trip. For a specific subject who is at risk, such a theoretical model would predict by how much each of these variables should be lowered to be certain of recovery when tripped.

In the present study, we therefore asked three questions related to during-step fallers. First, is the body orientation at the time that the recovery foot is lowered (tilt angle) critical for successful recovery? The second question was whether a simple inverted pendulum model, using subject-specific walking velocity and response time as input variables, could predict this body orientation, and thus success of recovery. The third question was whether reduction of walking velocity or reduction of response time would be more effective in preventing a fall after a trip.

\section{Materials and methods}

Data collection procedures and results have been described in detail elsewhere (Pavol et al., 1999a). Older adults ( $\geqslant 65 \mathrm{yr}$ of age, $n=79$ ) who were wearing an instrumented safety harness were unexpectedly tripped during locomotion. The experimental methods were reviewed and approved institutionally and each subject provided written informed consent prior to participation. A fall was marked by $>50 \%$ of the subject's body weight being supported by the safety harness. About half of the subjects used a "lowering strategy" for recovery, which consists of lowering the tripped foot to the ground and subsequently using the contralateral foot to step over the obstacle. Of those subjects who used a lowering strategy, eight falls and 26 recoveries were unambiguously identified. Only these 34 subjects were included in the present analysis. Of the eight falls, five were categorized as during-step falls, i.e. a fall occurred within $80 \mathrm{~ms}$ of the tripped foot being lowered to the ground, and three were categorized as after-step falls, i.e. the fall took place more than $470 \mathrm{~ms}$ after ground contact of the tripped foot.

For each subject, we defined response time, $T_{\mathrm{R}}$, as the elapsed time between the instant of the trip and the time at which the lowered foot contacted the ground. The latter was identified by a force plate signal sampled at $1 \mathrm{kHz}$. Sagittal plane body orientation $\theta$, determined from $60 \mathrm{~Hz}$ video data (Motion Analysis, Santa Rosa, $\mathrm{CA}$ ), was defined as the angle from the vertical, of the segment from the stance foot ankle joint to the center of mass of the body. The tilt angle, or body orientation at time of contact of the recovery foot, was determined by linear interpolation between video samples to determine $\theta$ at $t=T_{\mathrm{R}}$.

Subjects were modeled as a rigid body with mass $m$, moment of inertia $I$, and center of mass located at a distance $d$ from the stance from ankle joint. It was assumed that before the trip the body was moving forward with velocity $v$, forward tilt angle $\theta_{0}$ and zero angular velocity (Fig. 1). It was further assumed that following the trip, the body rotated about a fixed axis represented by the stance foot ankle joint. The change in linear and angular velocity was assumed to occur in an infinitesimally short time and, therefore, associated with an impulsive ground contact force (Hatze and Venter, 1981). Unknown variables are the horizontal ground reaction impulse $p$, the angular velocity $\omega_{0}$, and the horizontal velocity $v_{2}$ of the center of mass immediately after the trip. The values for the unknowns were solved from the impulse-momentum relationships

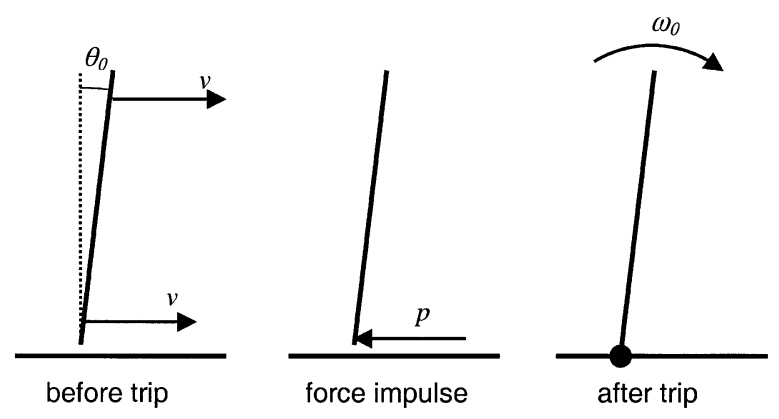

Fig. 1. Inverted pendulum model of the tripping event. Before the trip, the body moves forward with speed $v$, tilt angle $\theta_{0}$, and zero angular velocity. During the trip, assumed to have zero duration, a horizontal impulse $p$ is applied. Immediately afterwards, the body rotates with initial angular velocity $\omega_{0}$ about the ankle of the stance leg. 
for translation (Eq. (1)) and rotation (Eq. (2)), and a kinematic relationship between $\omega_{0}$ and $v_{2}$, assuming a constant center of rotation (Eq. (3)):

$m\left(v-v_{2}\right)=p$,

$I \omega_{0}=p d \cos \theta_{0}$,

$v_{2}=\omega_{0} d \cos \theta_{0}$,

Solving for the angular velocity gives

$\omega_{0}=\frac{m v d \cos \theta_{0}}{I+m d^{2} \cos ^{2} \theta_{0}}$.

If the subject is modeled as a uniform rod with length $h$, we can substitute $d=h / 2$ and $I=m h^{2} / 12$, and the only anthropometric parameters required are body height and mass. This simplifies Eq. (4) to

$\omega_{0}=\frac{6 v \cos \theta_{0}}{h\left(1+3 \cos ^{2} \theta_{0}\right)}$.

Therefore, the forward angular velocity immediately after the trip is proportional to the ratio between walking velocity and body height, independent of mass and only weakly dependent on the initial body orientation. Following the trip it was assumed that the body fell as an inverted pendulum with initial orientation $\theta_{0}$ and initial angular velocity $\omega_{0}$. For small rotations, the inverted pendulum motion is

$\theta(t)=\theta_{0} \cosh (k t)+\frac{\omega_{0}}{k} \sinh (k t)$

with

$k=\sqrt{\frac{m g d}{I+m d^{2}}}=\sqrt{\frac{3 g}{2 h}}$,

where $g$ is the acceleration of gravity. Walking velocity, body height, and initial angle are thus the only variables required to predict the movement of the inverted pendulum model after the trip.

The model was validated by comparing the predicted pendulum kinematics to the experimentally derived kinematics of the subjects who used a lowering strategy following the induced trip. Body height, walking velocity (of the body center of mass) prior to the trip, and initial tilt angle were measured for each subject and input into the model. The model predicted the body orientation between $t=0$ and $T_{\mathrm{R}}$, using Eq. (6). Predicted orientations were compared to the corresponding measured kinematics. Validation was not possible for three subjects due to incomplete data, two who recovered and one after-step faller. The relationship between the predicted and measured tilt angle $\theta_{\mathrm{R}}$, i.e. the body orientation at $t=T_{\mathrm{R}}$ in the remaining 31 subjects was quantified using the Pearson product-moment correlation.

The model was used to predict the extent to which tilt angle is dependent on walking velocity and response time for a hypothetical subject with body height of $1.7 \mathrm{~m}$ and an $8^{\circ}$ initial body orientation. These values approximate the means for the 34 adults tested. Determining the relative efficacy of reduction in walking velocity versus reduction in response time, was achieved by three simulation experiments on this hypothetical subject:

(1) If the subject has the average walking and response time of all 34 subjects, what is the effect on tilt angle of a one standard deviation decrease in walking velocity, versus a one standard deviation decrease in response time?

(2) If the subject has the average walking velocity and response time of the five during-step fallers, what is the effect of decreasing walking velocity to the population average, versus the effect of decreasing the response time to the population average?

(3) What is the "safe" walking velocity of a subject with the average response time of all during-step fallers, and by how much would this safe walking velocity increase if the subject had a normal response time?

\section{Results}

Measured tilt angle at time of foot contact $\left(\theta_{R}\right)$ was a perfect predictor of a during-step fall following the trip in this group of 34 subjects (Fig. 3, horizontal axis). Among the five during-step fallers, the lowest measured $\theta_{\mathrm{R}}$ was $26.3^{\circ}$ while among the 29 who successfully recovered or were after-step fallers the largest measured $\theta_{\mathrm{R}}$ was 23.0. Therefore, a threshold angle of between $23^{\circ}$ and $26^{\circ}$ separates the during-step fallers from those who performed a successful recovery step.

The model accurately reproduced the experimentally observed falling motions of the older adults who employed a lowering response to the induced trip (Fig. 2). Typically, the model slightly underestimated the initial angular velocity and overestimated the angular acceleration of the falling movement. The correlation between predicted and measured tilt angle was 0.930 and the prediction error was $0.4 \pm 2.2^{\circ}$ (Fig. 3). Predicted and measured tilt angles were equally effective in separating during-step fallers from the subjects who recovered.

After validation, the model was used to produce a contour plot of tilt angle, and by inference, success of recovery, as a function of walking velocity and response time for a subject with typical anthropometry (Fig. 4).

Predicted tilt angle, and therefore, risk of falling, was more sensitive to typical variations in response time than to typical variations in walking velocity (Fig. 5). For the hypothetical subject with a walking velocity and response time equal to the average of all 34 subjects $(0.70 \pm 0.11$ body heights per second and $175 \pm 55 \mathrm{~ms}$, 


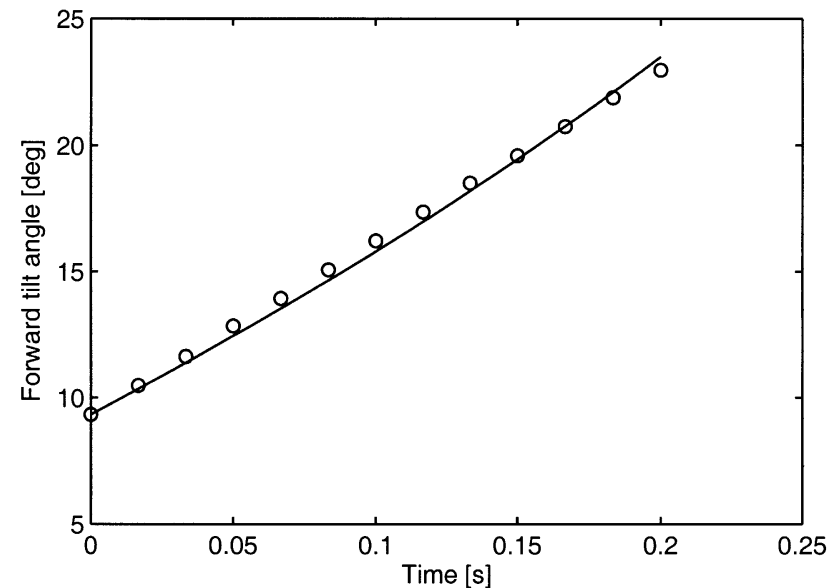

Fig. 2. Measured (open circles) and predicted rotation angle as a function of time for a typical subject (body height, $1.87 \mathrm{~m}$; walking velocity, 0.694 body heights per second; initial tilt, $9.3^{\circ}$; response time, $200 \mathrm{~ms})$.

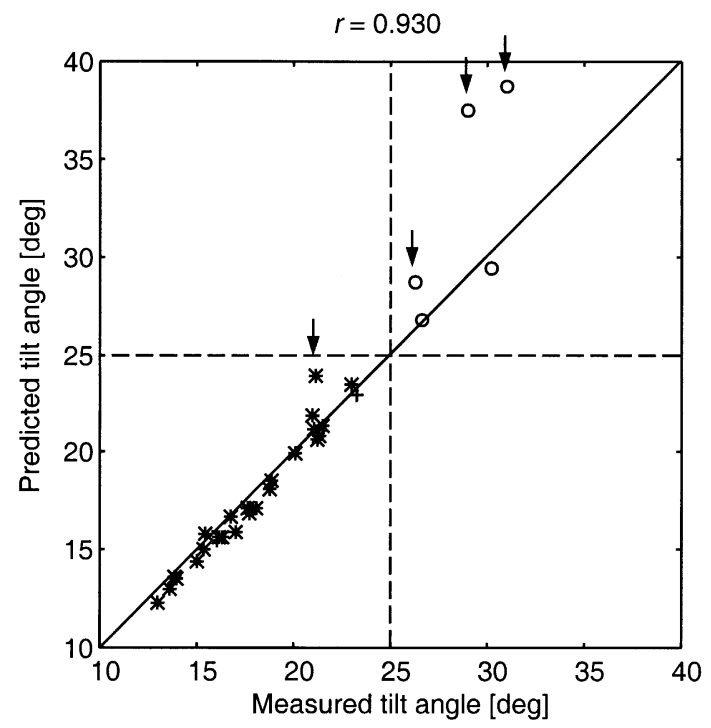

Fig. 3. Relationship between measured and predicted orientation of the body at the onset of weight bearing by the tripped foot. Arrows indicate subjects with a distinct deceleration of body rotation. A threshold body orientation of $25^{\circ}$ (dashed lines) perfectly separates the during-step fallers $(\bigcirc)$ from the subjects who recovered $(*)$ or fell after the step $(+)$.

respectively), the model predicted a tilt angle of $20^{\circ}$. A one standard deviation $(0.11 \mathrm{~h} / \mathrm{s})$ decrease in walking velocity resulted in a tilt angle of $18.3^{\circ}$. A one standard deviation $(55 \mathrm{~ms})$ decrease in response time resulted in a tilt angle of $15.9^{\circ}$. The larger sensitivity to changes of one standard deviation in response time, versus in walking velocity, was evident throughout the range of walking velocity and response times observed experimentally.

The model revealed that, for an average during-step faller, a normal response time would have been more

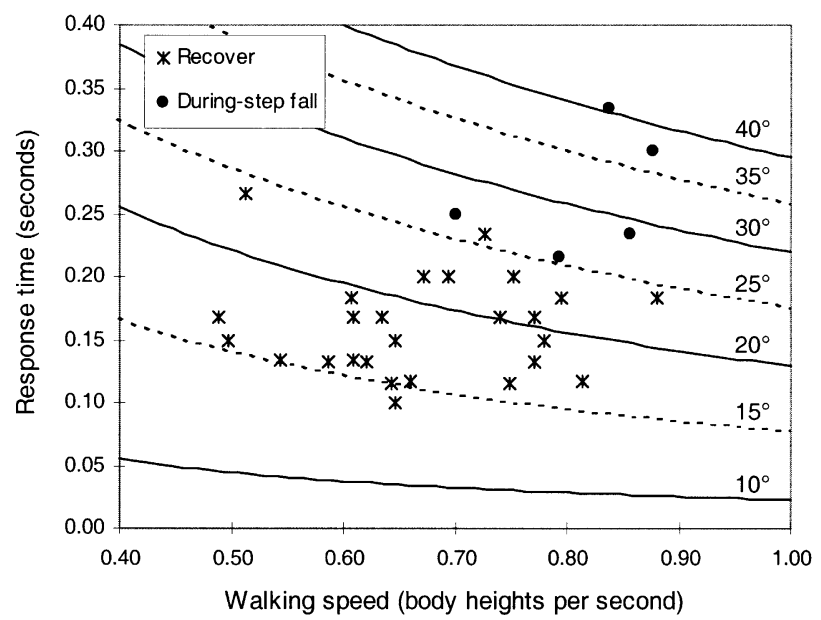

Fig. 4. Contour plot of body orientation at response time as a function of walking velocity for a typical subject with body height of $1.7 \mathrm{~m}$ and initial forward tilt of $8^{\circ}$. Superimposed are combinations of walking velocity and response time measured for the trips induced in the older adult subjects. Each subject's trip outcome is indicated. It should be noted that, due to variations in body height (range, $1.50-1.91 \mathrm{~m}$ ) and initial forward tilt angle (range, 5.4-10.7 ${ }^{\circ}$, the theoretical curves for the individual subjects would differ from those shown in Fig. 4.

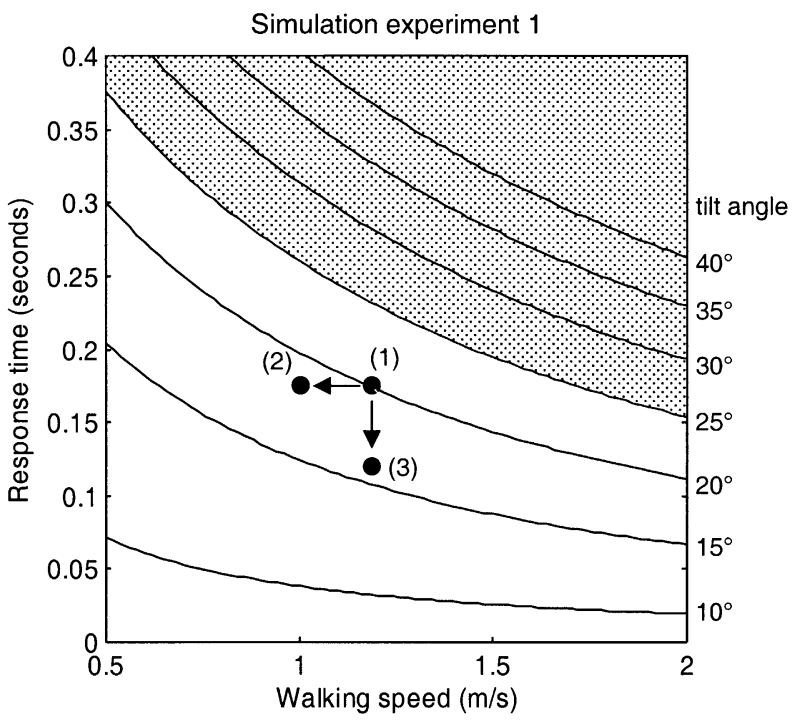

Fig. 5. Illustration of first model experiment. (1) Hypothetical subject with the average walking velocity and response time of all subjects. (2) Reduction of walking velocity by one standard deviation. (3) Reduction of response time by one standard deviation. Shaded area indicates where the model predicts a fall. The result indicates that variations in tilt angle, and thus trip outcome, arise mainly from variations in response time, rather than walking velocity.

effective at preventing a fall than would adopting a normal walking velocity (Fig. 6). The average duringstep faller had a walking velocity of $0.81 \pm 0.07$ body heights per second and a response time of $267 \pm 49 \mathrm{~ms}$. With these values, the model predicts an orientation of $31.3^{\circ}$ for the hypothetical subject at the time at which 


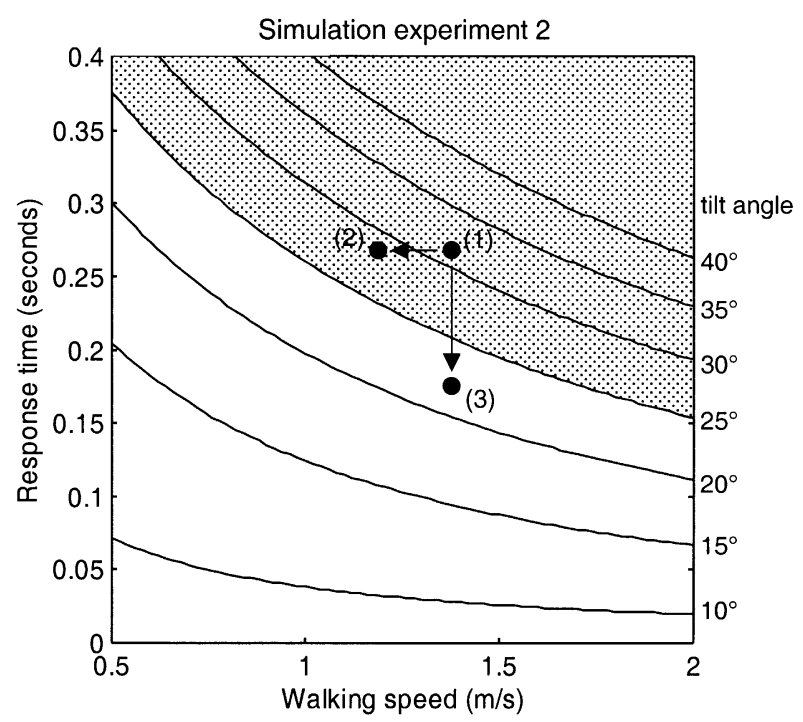

Fig. 6. Illustration of second model experiment. (1) Hypothetical subject with the average walking velocity and response time of all subjects who fell during the recovery step. (2) Reduction of walking velocity to the average of all subjects. (3) Reduction of response time to the average of all subjects. Shaded area indicates where the model predicts a fall. This result shows that abnormal reaction time had more influence on outcome of the trip than abnormal walking velocity.

the lowered foot contacted the ground. This is considerably above the $25^{\circ}$ limit for recovery. A normal walking velocity ( 0.70 body heights per second) reduced the tilt angle to $28.5^{\circ}$, which is still too large for recovery. A normal response time $(175 \mathrm{~ms})$ resulted in a tilt angle of $21.9^{\circ}$, which is well within the boundaries for successful recovery.

The model shows that decreased response time can substantially improve mobility of older adults who are at risk of falling (Fig. 7). For example, for the average during step faller with a response time of $267 \mathrm{~ms}$, the model predicts a safe walking velocity of 0.564 body heights per second $(0.96 \mathrm{~m} / \mathrm{s})$. However, response time of $175 \mathrm{~ms}$ allows safe walking velocity to increase to 1.0 body heights per second $(1.7 \mathrm{~m} / \mathrm{s})$.

\section{Discussion}

The present study was undertaken to predict the sensitivity of successful recovery after a trip to changes in walking velocity and response time in an older adult using a lowering strategy. A modeling approach was adopted because such causal relationships cannot be determined in human experiments due to inevitable adaptations and learning effects.

Before the model could make predictions about success of recovery, we had to test two additional hypotheses using human subject data. First, we showed

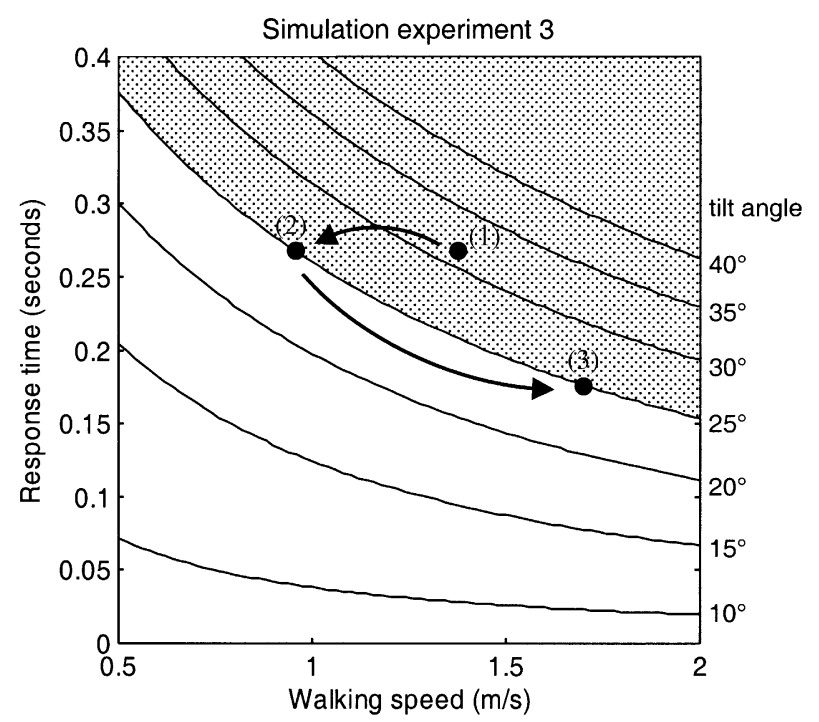

Fig. 7. Illustration of third model experiment. (1) Hypothetical subject with the average walking velocity and response time of the subjects who fell during the recovery step. (2) Reduction of walking velocity to the largest "safe" value predicted by the model. (3) Reduction of response time to the average of all subjects, followed by increase in walking velocity to the largest "safe" value predicted by the model. Shaded area indicates where model predicts a fall. This results shows that, in a typical person who is at risk for falling, safe walking velocity can almost be doubled when response time is improved.

that the recovery foot for a lowering strategy must contact the ground before the body tilt reaches a critical angle, which we found to be between $23^{\circ}$ and $26.3^{\circ}$. This is consistent with the maximum static lean angles from which older adults can recover (Wojcik et al., 1999), but it should be noted that this is a very different task. A limitation of our study is that the group was rather small. More experiments are needed to determine the extent to which there is overlap between the two groups. However, the $3^{\circ}$ of separation between 5 during-step fallers and the 26 others suggest that this overlap is small and that tilt angle is a predictor of a during-step fall after a trip. After-step falling does not seem related to the inverted pendulum mechanism (Fig. 3).

Second, we showed that our model correctly predicts the body tilt angle at time of recovery foot contact from an individual's walking velocity, response time, body height, and initial tilt angle. The model explained $87 \%$ of the variance in body tilt at the time of ground contact of the recovery foot. More importantly, tilt angle obtained with the model predicted the outcome of the recovery as effectively as the actual tilt angle. This justified the use of the model to explore the effects of walking velocity and response time on the outcome of the recovery after a trip. However, model limitations merit discussion because those will determine whether the model is still valid when used for situations not encountered in the human subject data used for validation. 
A limitation of the model was that the human body was simplified to a single rigid element with inertial properties of a slender rod with uniform density. Relative movement between body segments, which was not modeled, may explain why four of the subjects achieved lower tilt angles that predicted by the model, as indicated in Fig. 4. Incorporation of realistic mass distribution (Winter, 1979) did not improve the overall model predictions and was therefore not implemented.

A crucial assumption in the model was that the angular velocity of the body was zero just before the trip. We could have measured this variable, but this would have introduced another independent variable. Because of the cyclic nature of gait and left-right symmetry, there are four times during the gait cycle when the angular velocity of the entire body in the sagittal plane is exactly zero. Although it is unlikely that the trip occurred at one of those times, and angular velocity deviates considerably from zero between those times, predictions of the model were very good. To explain this, we propose that our "collision model", i.e. Eq. (5) for the initial angular velocity, includes the effect of all ground reaction forces that occurred since the last time when angular velocity was zero. This includes ground reaction forces on the stance leg, during that time interval, as well as the deceleration of the swing leg due to the obstacle. The fact that their combined effect could be predicted from inertial properties of a passive model can be taken as an indication that walking can be regarded as a series of passive collisions with the ground, where the limbs act like spokes in a wheel and muscles mainly serve to internally stabilize the body rather than provide active propulsion and deceleration.

The final question was answered using three model experiments. The first two showed that the effect of a typical variation in response time is larger than the effect of a typical variation in walking velocity. In this context, the term "typical" relates to either the observed variations within the entire subject population (Fig. 5), or to the differences between the during-step fallers and the other subjects (Fig. 6). In the third model experiment, it was shown that older adults who are at risk for falling can achieve a dramatic increase in safe walking velocity by improving their response time. Lowering the response time from 267 (the average of during-step fallers) to $175 \mathrm{~ms}$ (the average of all 34 subjects) will allow an increase of $77 \%$ in safe walking velocity without a concomitant increase in the likelihood of falling after a trip (Fig. 7). All three experiments suggested that response time is more important than walking velocity for the outcome of a trip.

The response time measured in this study is a combination of many neuromechanical processes including the time for the sensory system to detect the trip, to reflexively activate the relevant muscles, and the time required for the tripped leg to execute the stepping response. From the standpoint of possible intervention, it is important to determine if the central processing systems are responsible for the observed differences in response time, or if through modifying gait patterns or training specific responses it is possible to decrease the elapsed time required for the transition from swing phase to stance phase in the tripped leg. EMG analysis could be an important contributor to further study of the neural component of response time, and musculoskeletal dynamics to study the stepping response.

Response time appears to be an important determinant of recovery from tripping when using the lowering strategy. This is consistent with known risk factors (Lord et al., 1994; Lord and Clark, 1996). Reduction of reaction time as an intervention is therefore an interesting possibility. A current intervention paradigm is exercise (Gardner et al., 2000), and improvements in reaction time were found after an exercise program (Lord et al., 1995). However, exercise also improved strength and balance (Lord et al., 1995) and walking velocity increased (Lord et al., 1996). Therefore, further research using different experimental designs is needed to quantify the protective effect of reaction time improvement alone and determine whether reaction time training in a task not involving tripping would carry over to the recovery from a trip.

We suggest caution in recommending slower walking to healthy older adults. Even though we found this to contribute to successful recovery, large reductions in speed would be needed (Fig. 7). At very low speeds, the metabolic cost of locomotion increases and fatigue may become a limiting factor (Martin et al., 1992). Furthermore, falls at lower walking velocity are potentially more dangerous because impact is more likely to occur on the hip (Smeesters et al., 2001).

The conclusions from this study are:

(1) When a lowering strategy is used to recover from a trip, the tripped foot must contact the ground before the forward body tilt reaches a value of $26^{\circ}$ in order to be successful.

(2) An inverted pendulum collision model successfully predicts the tilt angle, and hence the success of recovery, from walking velocity, response time, body height, and body orientation at the instant of the trip.

(3) Variations in response time are more important in determining the success of recovery than are variations in walking velocity.

\section{Acknowledgements}

This research was partially supported by grant AG10557 from the National Institutes of Health (to MDG). 


\section{References}

Bath, P.A., Morgan, K., 1999. Differential risk factor profiles for indoor and outdoor falls in older people living at home in Nottingham, UK. European Journal of Epidemiology 15, 65-73.

Cumming, R.G., Klineberg, R.J., 1994. Fall frequency and characteristics and the risk of hip fractures. Journal of the American Geriatrics Society 42, 774-778.

Dargent-Molina, P., Favier, F., Grandjean, H., Baudoin, C., Schott, A.M., Hausherr, E., Meunier, P.J., Breart, G., 1996. Fall-related factors and risk of hip fracture: the EPIDOS prospective study. Lancet 348, 145-149.

Gardner, M.M., Robertson, M.C., Campbell, A.J., 2000. Exercise in preventing falls and fall related injuries in older people: a review of randomised controlled trials. British Journal of Sports Medicine $34,7-17$.

Hatze, H., Venter, A., 1981. Practical activation and retention of locomotion constraints in neuromusculoskeletal control system models. Journal of Biomechanics 14, 873-877.

Hayes, W.C., Myers, E.R., Robinovitch, S.N., van den Kroonenberg, A., Courtney, A.C., McMahon, T.A., 1996. Etiology and prevention of age-related hip fractures. Bone $18,77 \mathrm{~S}-86 \mathrm{~S}$.

Lord, S.R., Clark, R.D., 1996. Simple physiological and clinical tests for the accurate prediction of falling in older people. Gerontology 42, 199-203.

Lord, S.R., Ward, J.A., Williams, P., Strudwick, M., 1995. The effect of a 12-month exercise trial on balance, strength, and falls in older women: a randomized controlled trial. Journal of the American Geriatrics Society 43, 1198-1206.

Lord, S.R., Ward, J.A., Williams, P., Anstey, K.J., 1994. Physiological factors associated with falls in older community- dwelling women. Journal of the American Geriatric Society 42, $1110-1117$

Lord, S.R., Lloyd, D.G., Nirui, M., Raymond, J., Williams, P., Stewart, R.A., 1996. The effect of exercise on gait patterns in older women: a randomized controlled trial. Journal of Gerontology: Medical Sciences 51A, M64-M70.

Martin, P.E., Rothstein, D.E., Larish, D.D., 1992. Effects of age and physical activity status on the speed-aerobic demand relationship of walking. Journal of Applied Physiology 73, 200-206.

Nyberg, L., Gustafson, Y., Berggren, D., Brännström, B., Bucht, G., 1996. Falls leading to femoral neck fractures in lucid older people. Journal of the American Geriatrics Society 44, 156-160.

Pavol, M.J., Owings, T.M., Foley, K.T., Grabiner, M.D., 1999a. Gait characteristics as risk factors for falling from trips induced in older subjects. Journal of Gerontology: Medical Sciences 54 A, M583-M591.

Pavol, M.J., Owings, T.M., Foley, K.T., Grabiner, M.D., 1999b. The sex and age of older adults influence the outcome of induced trips. Journal of Gerontology: Medical Sciences 54 A, M103-M108.

Pavol, M.J., Owings, T.M., Foley, K.T., Grabiner, M.D., 2001. Mechanisms leading to a fall from an induced trip in healthy older adults. Journal of Gerontology: Medical Sciences 56 A, M428-M437.

Smeesters, C., Hayes, W.C., McMahon, T.A., 2001. Disturbance type and gait speed affect fall direction and impact location. Journal of Biomechanics 34, 309-317.

Winter, D.A., 1979. Biomechanics of Human Movement. Wiley, New York.

Wojcik, L.A., Thelen, D.G., Schultz, A.B., Ashton-Miller, J.A., Alexander, N.B., 1999. Age and gender differences in single-step recovery from a forward fall. Journal of Gerontology: Medical Sciences 54 A, M44-M50. 